\title{
Effect of Oxygen Doping on the Structure of TiN Surface Coatings
}

\author{
Domokos BÍRÓ $^{1 \mathrm{a}}$, László JAKAB-FARKAS ${ }^{1 \mathrm{~b}}$, András \\ KELEMEN $^{1 \mathrm{~b}}$, Sándor PAPP ${ }^{1 \mathrm{~b}}$, Mohamed Fathy HASANEEN ${ }^{2}$, Miklós \\ MENYHÁRD $^{2}$, Sándor GURBÁN ${ }^{2}$, Péter B. BARNA ${ }^{2}$ \\ ${ }^{1 a}$ Department of Mechanical Engineering, Faculty of Technical and Human Sciences, \\ Sapientia University, Tg. Mureş, e-mail: $\underline{\text { dbiro@ms.sapientia.ro }}$ \\ ${ }^{1 b}$ Department of Electrical Engineering, Faculty of Technical and Human Sciences, \\ Sapientia University, Tg. Mureș, e-mail: jflaci@ms.sapientia.ro, \\ kandras@ms.sapientia.ro, spapp@ms.sapientia.ro \\ ${ }^{2}$ Institute for Technical Physics and Materials Science, Centre for Energy Research, Hungarian \\ Academy of Sciences, H-1525 Budapest, Po Box 49, Hungary
}

Manuscript received January 12, 2015, revised February 9, 2015.

Abstract: In the present work the influence of the level of oxygen doping on the structure of TiN films was investigated by dedicated experiments. The films were deposited at $400^{\circ} \mathrm{C}$ in an all metal UHV device by unbalanced magnetron sputtering at the same Ar and nitrogen flow rates, but the oxygen flow rate was changed in the experiments, incorporating oxygen in the range of 4 and 20 at.\%. The structure of the films was investigated by XRD, Auger electron (AES) and X-ray photon electron (XPS) spectroscopy and transmission electron microscopy (TEM). The results discovered the crystal face anisotropy in the incorporation-segregation of oxygen leading to the change of the $<111>$ texture to $<002>$. The structure analysis revealed that the $<002>$ texture is developing also by competitive growth of crystals, which is the result of the limitation of the growth of the $<111>$ oriented crystals by the $\mathrm{TiO}_{2}$ layer developing on their growth surface by the segregated oxygen species. The oxygen incorporating in the crystal lattice on the 002 crystal faces of the $<002>$ oriented crystals is segregated by surface spinodal decomposition, developing $\mathrm{nm}$ sized $3 \mathrm{D} \mathrm{TiO}_{2}$ inclusion both in the bulk of the columns and the column boundaries.

Keywords: $\mathrm{TiO}_{\mathrm{x}} \mathrm{N}_{\mathrm{y}}$ thin films, texture, XTEM, XRD, AES, XPS.

\section{Introduction}

TiN thin films are applied preferentially as protective or hard surface coatings on tools and component parts on various instruments, including also the devices used in mechatronics. Their structure and properties are sensitively controlled by doping materials. Beside the most frequently used $\mathrm{Si}$ and $\mathrm{Al}$, oxygen is playing an important role in tailoring the structure and by this way the mechanical and chemical properties. 
It is generally experienced that by doping the TiN film by oxygen the structure is modified.The texture changed from $<111>$ to $<001>$ with increase of oxygen concentration: the $<111>$ texture changed to $<001>$ if the oxygen concentration was higher than 15 at.\%[1-3]. Ehiasarian et al. reported on the development of $<001>$ texture in TiN film deposited by high power impulse magnetron sputtering (HIPIMS)[4]. In these experiments the deposition started at high oxygen partial pressure (as contamination related to the degassing of the system) incorporating $\sim 18$ at.\% oxygen and after the degassing, as the oxygen pressure decreased, the concentration of incorporated oxygen is decreased to 2 at.\%. The volume fractions of crystals at the surface of the film with random $<001>$ and $<111>$ orientations were $10 \%, 64 \%$ and $26 \%$, respectively. These indicated that the development of the $<001>$ texture started but had not completed at this film thickness. Considering the V-shaped morphology of the $<001>$ oriented single crystal columns Ehiasarian et al. suggested that the $<001>$ texture could be developed by competitive growth[4]. However, the mechanisms which could control the competitive growth at oxygen doping beyond a critical level has not been completely clarified yet.

In the present work structural information has been collected on oxygen doped $<111>$ and $<001>$ oriented TiNfilms which could validate the competitive nature of the growth of crystals in both cases responsible for the texture development. Only films with homogenous oxygen concentration distribution along the thickness have been considered. In the present paper results on selected samples are reported.

\section{Experimental details}

Titanium nitride thin films with thicknesses around $1.3 \mu \mathrm{m}$ were deposited in a home made laboratory scale magnetron sputter unit [5] at $400^{\circ} \mathrm{C}$ substrate temperature, at $0.2 \mathrm{nms}^{-1}$ deposition rate on $20 \times 20 \mathrm{~mm}^{2}<001>$ oriented Si single crystal substrates covered by native oxide. The octagonal all-metal high vacuum chamber of 75 litre volume was evacuated by a 540 1/s turbo-molecular pump producing $2 \times 10^{-4} \mathrm{~Pa}$ background pressure. The magnetic fields of closely disposed magnetron sources arranged on an arc segment were highly interacting, leading to a far extended active plasma volume arriving to the substrate. A planar rectangular metallic Ti target $\left(165 \times 85 \times 12 \mathrm{~mm}^{3}\right.$ in size $)$ of $99.95 \%$ purity (PLANSEE GmbH) was used. The discharge plasma was excited reactively in a mixture of $\mathrm{Ar}, \mathrm{N}_{2}$ and $\mathrm{O}_{2}$ by a close-loop controlled de power supply. The discharge power for each deposition run was $500 \mathrm{~W}$. The Ar gas flow rate was stabilized by a solenoid valve actuated mass flow controller (MFC-Granville Phillips S 216), and measured by a GFM 17 Aalborg mass flow meter. The nitrogen flow rate was controlled by Aalborg DFC 26 digital 
mass flow controller. Introduction of oxygen gas was manually controlled by using a high precision needle valve (Granville Phillips type).

$\mathrm{X}$-ray diffraction (XRD) and transmission electron microscopy (TEM) techniques were used for the analysis of the microstructure of the films. A Bruker AXS D8 Discover diffractometer equipped with Göbel-mirror and a 2D position sensitive (GADDS) detector system with $\mathrm{Cu} \mathrm{K} \alpha$ radiation were used for XRD analysis. The intensity was acquired at four detector positions at nominal $2 \theta$ angles of $20^{\circ}, 40^{\circ}, 60^{\circ}$ and $80^{\circ}$, covering the range of about $7^{\circ}-$ $93^{\circ}$, while the samples were positioned at $\theta=10^{\circ}, 20^{\circ}, 30^{\circ}$ and $40^{\circ}$, respectively. In the present diffraction patterns the Si 400 reflection is not shown because the measurements have been carried out at sample position $\theta=30^{\circ}$ and not at $\theta=34.56^{\circ}$.

Cross-sectional (X-TEM) and plan-view specimens were prepared by mechanical cutting, grinding and ion beam thinning techniques for TEM investigations [6, 7]. A Philips CM20 transmission electron microscope working at $200 \mathrm{kV}$ and equipped with a Ge-detector NORAN EDX analyzer was applied for the conventional TEM investigation and chemical analysis. The high-resolution TEM investigation was carried out in a $300 \mathrm{kV}$ JEOL 3010 TEM. Selected area electron diffraction (SAED) was applied to analyze the variation of the texture along the thickness of the film. The "ProcessDiffraction" software $[8,9]$ made possible both the qualitative and quantitative evaluation of the selected area electron diffraction patterns. Reflection high energy electron diffraction (RHEED) made it possible to determine the orientation of crystals penetrating to the surface of the film.

The chemical composition and its variation along the thickness of the films were investigated by Auger electron spectroscopy (AES) and X-ray photon electron spectroscopy (XPS) depth profiling. Sample rotation and glancing angle ion bombardment techniques were applied during depth profiling; the atomic concentrations were calculated by applying the sensitivity factor (RSF) method. In case of the XPS spectra the background was removed by the Shirley method and the following RSF parameters were used: 1.8 for Ti2p, 0.42 for N1s and 0.66 for O1s $[10,11]$.

\section{Results}

\subsection{Structure of the coating incorporated 4 at.\% oxygen}

\subsubsection{TEM and SAED analyses}

Fig. 1 shows the cross-sectional bright field transmission electron microscopic (X-TEM) image of the TiN film containing 4 at.\% oxygen, while the selected area diffraction patterns taken from the various areas of the film along the thickness are given in the insets a-f. According to the X-TEM image 
the film exhibits the characteristic zone $\mathrm{T}$ structure of $\mathrm{V}$-shaped columns [12, 13].The crystal size is initially small and increases continuously with film thickness due to the $\mathrm{V}$-shaped morphology of columns. The column tops are faceted. The reflection high energy electron diffraction pattern (inset $g$ in Fig. 1) indicates that the crystal columns nucleated on the substrate proceeded to the surface of the film are $<111>$ oriented.

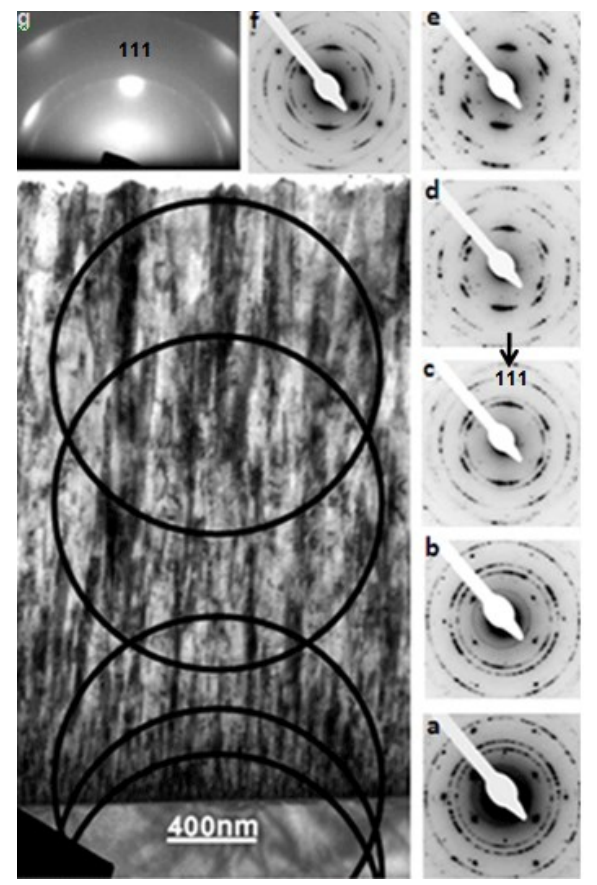

Figure 1. Cross sectional TEM image and SAED patterns of the TiN film containing 4 at.\% oxygen. SAED patterns were taken from the areas marked by circles.

The SAED pattern taken from an area including both the substrate and the whole cross section of the film (inset $f$ in Fig. 1) confirms the existence of $<111>$ texture identified also by XRD. The strong 111 reflection is in the direction of the 001 reflection of Si single crystal substrate and proves clearly that the texture axis is parallel to the substrate surface normal, the $<001>$ direction of the Si single crystal substrate, in agreement with the direction of column's axes. The SAED patterns taken successively along the film thickness clearly demonstrate the successive evolution of the $<111>$ texture. The small crystals developed on the substrate by nucleation are randomly oriented (inset a). Intensity of the 111 reflection in the direction of the substrate surface normal is increasing with film thickness (insets $b, c$ and $d$ ). The simultaneous 
development of the V-shaped morphology of the $<111>$ oriented crystals nucleated on the substrate can be clearly observed. The SAED pattern of the upmost part of the film (Fig. 1 inset e) shows already a complete $<111>$ texture in accordance with the RHEED pattern (Fig. 1 inset g).

The N/Ti ratio in the bulk determined by XPS was 1.12 and the film contained 4 at.\% oxygen as bulk contamination. The $\mathrm{N} / \mathrm{Ti}$ ratio and the concentration of oxygen were homogeneous in the film.

\subsubsection{X-ray diffraction analysis}

The XRD spectrum of the undopedTiN film containing 4 at.\% oxygen, shown in Fig. 2a, agrees well with the cubic TiN structure (JCPDS card number 38-1420). The spectrum contains traces of 002 and 220 reflections in addition to the strong 111 and 222 ones, indicating presence of the randomly oriented crystals developed on the substrate in the first stage of film growth, as indicated already by the SAED pattern (inset a in Fig. 1). The XRD confirmed the strong $<111>$ fiber texture with a texture coefficient $\mathrm{TC} \approx 1$, in agreement with the SAED pattern of whole cross section of the film (inset $g$ in Fig. 1). The size of coherent scattering domains calculated from line broadening of 111 and 222 XRD reflections $[14,15]$ was $6.2 \mathrm{~nm}$ not taking into account the instrumental broadening of the diffractometer.

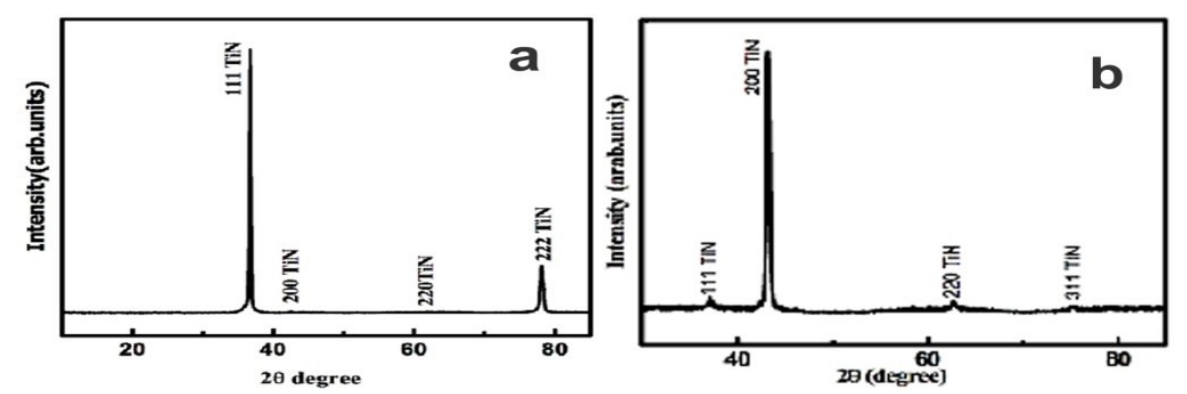

Figure 2. XRD diffraction patterns of TiN films containing different concentration of oxygen: a) 4 at.\%; b) 20 at.\%.

\subsection{Structure of the coating incorporated 20 at.\% oxygen}

\subsubsection{XRD analysis}

The XRD pattern of the film is shown in Fig. $2 \mathrm{~b}$. This pattern contains a strong 200 reflection, while the intensity of the 111, 220 and 311 reflections are very weak. These indicate that the film is grown with $<001>$ texture but contains again randomly oriented crystals.

\subsubsection{TEM and SAED analyses}


The cross-sectional bright field TEM image of the TiN film doped with 20 at.\% oxygen is shown in Fig. 3. This film exhibits also the characteristic zone $\mathrm{T}$ structure of $\mathrm{V}$-shaped columns as identified in the non-doped film shown in (Fig. 1). Accordingly the size of crystals developed by nucleation on the substrate is small and these crystals are randomly oriented producing the weak 111,220 and 311 reflections present in the XRD pattern. Crystals with the preferred $<111>$ orientation are grown in V-shaped columns. The column tops are with shallow cup-shape morphology, resulting in a low surface roughness.

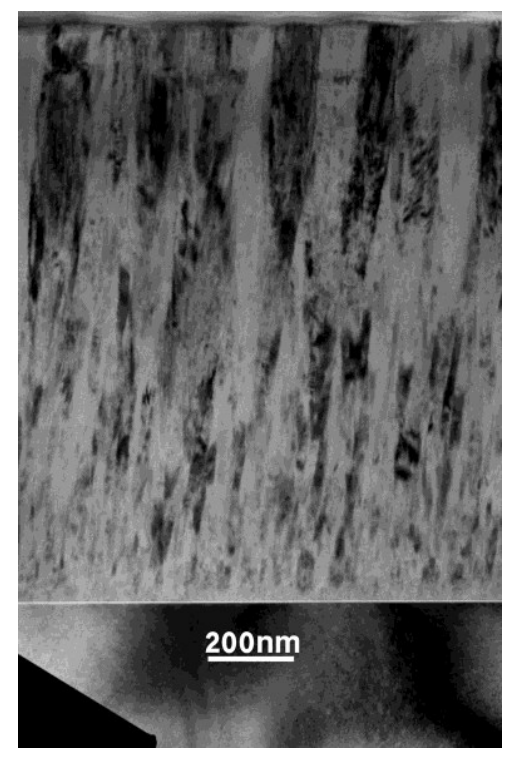

Figure 3. Cross sectional TEM image of the TiN sample containing 20 at. $\%$ oxygen

The high resolution phase contrast TEM image of columns reveals a substructure of the columnar crystals [16]. This substructure is well detectable both in the cross sectional and plan view specimens. The bulk of the columnar single crystals contains necklace-like chains of fine grains showing up in white fibres in the phase contrast X-TEM image (Fig. 4a). In the plane view specimen, containing the cross section of the columns, it is clearly to be seen that these fibres are arranged in a honeycomb-like structure (Fig. 4b). The analysis of the selected area diffraction pattern of the film, shown in Fig. 5, proves that beside the c-TiN phase (JCPDS card: No.38-1420) the tetragonal $\mathrm{TiO}_{2}$ phase (JCPDS card: No.82-0514) (110 diffraction spot marked by an arrow) is also present. Considering the sharp diffraction line of the TiN phase, one can conclude that the size of TiN crystals is large, while the size of $\mathrm{TiO}_{2}$ 
crystals can be in the nanometer range due to the diffuse reflection. One can also conclude that the crystals of both phase are oriented (the film is textured with $<001>\mathrm{TiN}$ ), and the $\mathrm{TiN}$ and $\mathrm{TiO}_{2}$ crystals are epitaxially related $\left(\mathrm{TiN}<001>/ / \mathrm{TiO}_{2}<110>\right)$. The $\mathrm{TiO}_{2}$ nanocrystals are with 3D morphology.

The chemical composition and the volume fraction of phases have been determined by XPS analysis. Accordingly the $65 \%$ of oxygen incorporated into the films was dissolved in the crystal lattice, while $35 \%$ was present in $\mathrm{TiO}_{2}$ segregates.
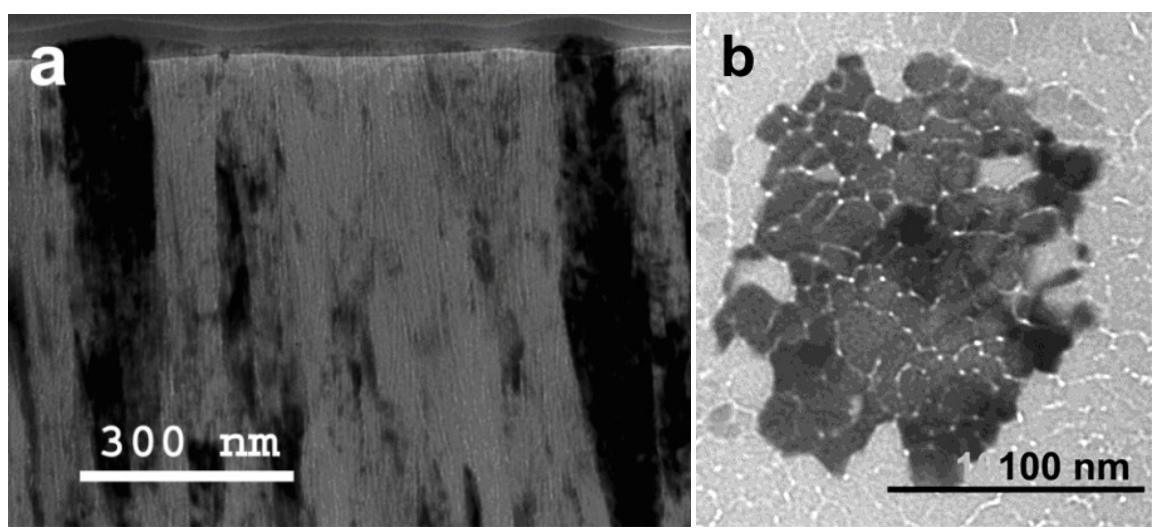

Figure 4. The phase contrast X-TEM image (a) and plan view TEM image of TiN coating containing 20 at.\% oxygen show the distribution of $3 \mathrm{D} \mathrm{TiO}_{2}$ segregated phase.

$\mathrm{TiO}_{2}$ segregates are shown in white contrast.

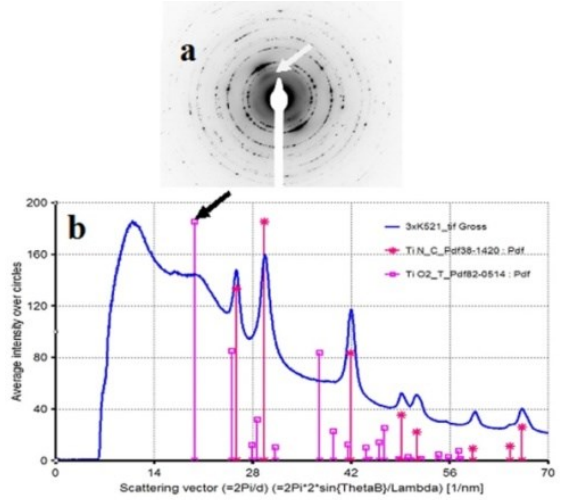

Figure 5.Selected area electron diffraction pattern of the TiN coating containing 20 at.\% oxygen. The $\mathrm{TiO}_{2} 110$ reflections are marked by arrows.

\subsection{Structure of the coating incorporated 11 at.\% oxygen}


Preliminary XRD and X-TEM investigations of the film revealed that the film was grown with columnar structure according to the zone $\mathrm{T}$ structure and the texture is strong $<111>$. According to the XPS analyses no oxygen has been dissolved into the crystal lattice but it was present in $\mathrm{TiO}_{2}$ phase. The phase contrast TEM images of the cross sectional specimens revealed a very detailed, fragmented bulk structure of the columnar TiN crystals shown in Fig. 6. The columnar crystals are constituted of lamellar-like domains ordered in subcolumns separated by a foreign phase showing up clearly as white lines in the phase contrast images. This thin inter-domain layer can be attributed to the $\mathrm{TiO}_{2}$ phase.

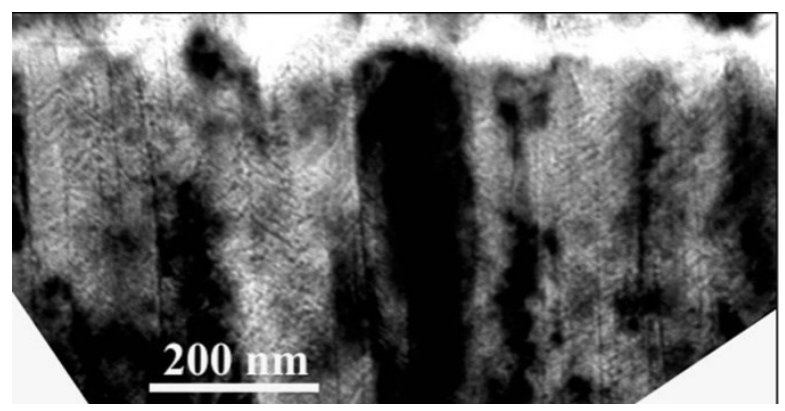

Figure 6. Phase contrast X-TEM image of the TiN coating containing 11 at.\% oxygen.

\section{Discussion and conclusions}

By analyzing the X_TEM images one can conclude that the structure of the all three coatings with increasing oxygen concentration is characterized by the zone $\mathrm{T}$ structure. This structure develops by a pathway of structure evolution constituted from three characteristic growth regimes developing three kind of structures in well distinguished thickness ranges $[12,13,17]$. This pathway is characterized by the competitive growth of crystals controlled by the dependence of the growth rates on the various crystal faces. According to the present results, the growth rate is the largest on the $<111>$ crystal faces up to a given concentration of oxygen (about 15 at. $\%$ ) at $400^{\circ} \mathrm{C}$ substrate temperature. While at 20 at.\% oxygen concentration an other effect has to control the growth rate of crystals on the various faces.

The phase contrast TEM images revealed that the interaction of oxygen on the different crystal faces and the growth mode of the $\mathrm{TiO}_{2}$ phase are different in case of crystals growing with $<111>$ and $<001>$ orientation. In case of $<111>$ oriented crystals, where the growth crystal faces are the 111, the oxygen is completely segregated by the surface atomic processes (kinetic segregation). 
The segregated oxygen nucleates the $\mathrm{TiO}_{2}$ phase, which is growing in 2D layer at the columns boundaries and on the growth surface. Consequently, this $2 \mathrm{D}$ surface covering oxide layer will cover the growth surface and limits the further incorporation of Ti, i.e. the growth of $<111>$ oriented crystals. While in case of crystals with $<001>$ orientation on the 001 crystal faces absorb (dissolve) a large part of impinging oxygen species and the excess oxygen species form $3 \mathrm{D}$ $\mathrm{TiO}_{2}$ segregates incorporated into the bulk of TiN crystals. These do not limit the growth of the $<001>$ oriented crystals, consequently at high oxygen concentration (e.g 20 at.\%) the $<001>$ oriented crystals will win the competitive growth developing a coating with columns of V-shaped morphology and with $<001>$ texture. That is the same mechanism as discovered and described in case of oxygen doped Al thin films [12, 13,17].

\section{Acknowledgements}

D.B., L. J.-F., P.S. and K.A. would like to appreciate the funding by the HungarianAcademy of Sciences, in frame of Hungarian Science AbroadFellowship Program.

\section{References}

[1] Makino, Y., et al., Characterization of Ti(NxOy) coatings produced by the arc ion plating method. Surface and Coatings Technology, 1998. 98(1-3): p. 934-938.

[2] Chan, M.-H. and F.-H. Lu, Preparation of titanium oxynitride thin films by reactive sputtering using air/Ar mixtures. Surface and Coatings Technology, 2008. 203(5-7): p. 614-618.

[3] Wu, Y., et al., Microstructure and mechanical properties of reactively sputtered $\mathrm{Ti}(O, N)$ coatings. International Journal of Refractory Metals and Hard Materials, 2008. 26(5): p. 461-464.

[4] Ehiasarian, A.P., et al., Influence of high power impulse magnetron sputtering plasma ionization on the microstructure of TiN thin films. Journal of Applied Physics, 2011. 109(10): p. 104314.

[5] Jakab-Farkas, L., S. Papp, and D. Biro. Effect of $N$ Concentration on Microstructure Evolution of the Nanostructured (Al, Ti, Si)N Coatings Prepared by d.c. Reactive Magnetron Sputtering. in Proceedings of the 3rd International Conference Inter-Eng 2009, Interdisciplinarity in Engineering. 2009.

[6] Barna, Á., Topographic Kinetics and Practice of Low Angle Ion Beam Thinning. MRS Online Proceedings Library, 1991. 254: p. null-null.

[7] Barna, Á., B. Pécz, and M. Menyhard, TEM sample preparation by ion milling/amorphization. Micron, 1999. 30(3): p. 267-276.

[8] Labar, J.L., Electron Diffraction Based Analysis of Phase Fractions and Texture in Nanocrystalline Thin Films, Part II: Implementation. Microscopy and Microanalysis, 2009. 15(1): p. 20-29.

[9] Lábár, J.L., Consistent indexing of a (set of) single crystal SAED pattern(s) with the ProcessDiffraction program. Ultramicroscopy, 2005. 103(3): p. 237-249. 
324 D. Bíró, L. Jakab-Farkas, A. Kelemen, S. Papp, M.F. Hasaneen,M. Menyhárd, S.Gurbán, P.B. Barna

[10] Briggs, D. and P. Seah, Practical Surface Analysis, Auger and X-ray Photoelectron Spectroscopy. 1990: Wiley.

[11] Guillot, J., et al., Amorphous TiO2 in LP-OMCVD TiNxOy thin films revealed by XPS. Applied Surface Science, 2001. 177(4): p. 268-272.

[12] Barna, P.B. and M. Adamik, Fundamental structure forming phenomena of polycrystalline films and the structure zone models. Thin Solid Films, 1998. 317(1-2): p. 27-33.

[13] Petrov, I., et al., Microstructural evolution during film growth. Journal of Vacuum Science \&amp; Technology A, 2003. 21(5): p. S117-S128.

[14] Xiao, L., et al., Nanostructured TiN coating prepared by reactive plasma spraying in atmosphere. Applied Surface Science, 2007. 253(18): p. 7535-7539.

[15] Gubicza, J., et al., Influence of sintering temperature and pressure on crystallite size and lattice defect structure in nanocrystalline SiC. Journal of Materials Research, 2007. 22(05): p. 1314-1321.

[16] Hasaneen, M.F., et al., Substructure in the columnar crystals of the Ti0.45O0.20N0.35 oxynitride thin film. Vacuum, 2012. 86(12): p. 2105-2108.

[17] Barna, P.B. and G. Radnóczi, 3 - Structure formation during deposition of polycrystalline metallic thin films, in Metallic Films for Electronic, Optical and Magnetic Applications, K. Barmak and K. Coffey, Editors. 2014, Woodhead Publishing. p. 67-120. 